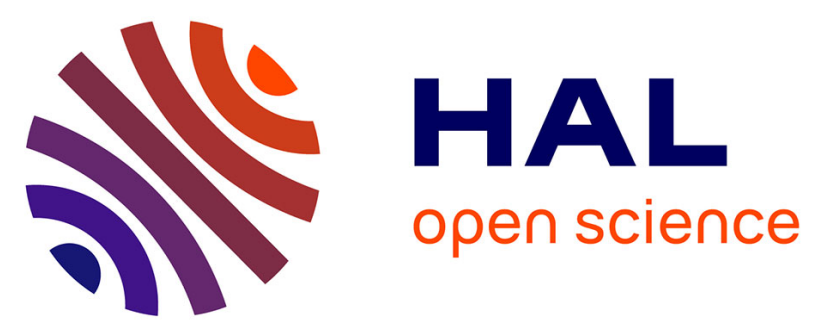

\title{
Dynamique géomorphologique holocène et occupation humaine dans le bassin-versant de l'Eure (Bassin de Paris, France) : potentiels d'une approche géoarchéologique multiscalaire et diachronique
}

Théophile Piau, Francois Betard, Fabienne Dugast, Gilles Arnaud-Fassetta, Vincent Viel

\section{To cite this version:}

Théophile Piau, Francois Betard, Fabienne Dugast, Gilles Arnaud-Fassetta, Vincent Viel. Dynamique géomorphologique holocène et occupation humaine dans le bassin-versant de l'Eure (Bassin de Paris, France): potentiels d'une approche géoarchéologique multiscalaire et diachronique. Archimède: archéologie et histoire ancienne, 2020, 7, pp.188-204. halshs-02893624

\section{HAL Id: halshs-02893624 \\ https://shs.hal.science/halshs-02893624}

Submitted on 8 Jul 2020

HAL is a multi-disciplinary open access archive for the deposit and dissemination of scientific research documents, whether they are published or not. The documents may come from teaching and research institutions in France or abroad, or from public or private research centers.
L'archive ouverte pluridisciplinaire HAL, est destinée au dépôt et à la diffusion de documents scientifiques de niveau recherche, publiés ou non, émanant des établissements d'enseignement et de recherche français ou étrangers, des laboratoires publics ou privés. 


\section{ARCHIMÈDE N N 7}

\section{DOSSIER THÉMATIQUE : GESTES RITUELS. DE LA TRACE À L'INTERPRÉTATION}

\section{ACTUALITÉ DE LA RECHERCHE GÉOSCIENCES ET ARCHÉOLOGIE : INTERACTIONS, COMPLÉMENTARITÉS ET PERSPECTIVES}

114 Bruno GAVAZZI

Heureuses rencontres. Vers le développement d'approches intégrées en géosciences et archéologie

119 Jean-Paul BRAVARD

Dialogue interdisciplinaire : de I'unité stratigraphique aux interactions culture-environnement

129 Ferréol SALOMON

Les origines d'Ostie : quelles interactions avec la dynamique d'embouchure? (Delta du Tibre, Italie)

141 Quentin BORDERIE, Rowena Y. BANERJEA, Stéphane BONNET, Yannick DEVOS, Cristiano NICOSIA, Christophe PETIT, Ferréol SALOMON, Nathalie SCHNEIDER, Barbora WOUTERS, \& Patrice WUSCHER Géoarchéologies des contextes urbains : mieux comprendre les modalités de l'artificialisation des géosystèmes

158 Patrice WUSCHER, Christophe JORDA, Quentin BORDERIE, Nathalie SCHNEIDER \& Laurent BRUXELLES De la formation géologique à la tranchée : trouver et comprendre les sites archéologiques menacés par les travaux d'aménagement du territoire

176 Morgan MILLET \& Michel GUÉLAT

Les vestiges antiques de Rennaz-Noville (Vaud, Suisse) et leur contexte sédimentaire : nouvelles évidences de l'écroulement du Tauredunum

188 Théophile PIAU, François BÉTARD, Fabienne DUGAST, Gilles ARNAUD-FASSETTA \& Vincent VIEL Dynamique géomorphologique holocène et occupation humaine dans le bassin-versant de l'Eure (Bassin de Paris, France) : potentiels d'une approche géoarchéologique multiscalaire et diachronique

205 Dominique SCHWARTZ, Vincent ROBIN, Pierre ADAM, Philippe SCHAEFFER, Anne GEBHARDT, Pierre-Alexis HERRAULT, Benjamin KELLER, Daniele DAPIAGGI, Claire STEVENEL, Maxime THISS, Martine TRAUTMANN \& Damien ERTLEN

Les géosciences au service de l'archéologie agraire. Une étude de cas sur les rideaux de culture de Goldbach (68)

217 Étienne MANTEL, Stéphane DUBOIS, Jonas PARÉTIAS, Victor VISQUESNEL-SCHLOSSER, Corentin VOISIN, Bruno GAVAZZI \& Matthieu RICHARD

Étudier l'occupation d'une ville : les enjeux du PCR « Topographie générale et insertion territoriale de I'agglomération antique de Briga »

231 Charlène MOREL

Un modèle pour comprendre l'influence de l'état de la recherche, des processus post-déposition et de l'attractivité sur la découverte des sites archéologiques dans le Kochersberg (Bas-Rhin, Alsace, Grand-Est, France)

241 Lizzie SCHOLTUS

Spatialisation des découvertes, modélisation du passé. L'informatique au service de l'archéologie

254 Guillaume HULIN \& François-Xavier SIMON

Inrap et géophysique : vers une approche raisonnée

260 Hugo REILLER, Matthieu FUCHS, \& Bruno GAVAZZI

Approche multi-méthodes expérimentale pour l'étude d'un site d'occupation romaine et médiévale à Horbourg-Wihr

272 François-Xavier SIMON, Julien GUILLEMOTEAU, Guillaume HULIN, Joachim RIMPOT, Julien THIESSON \& Alain TABBAGH

De nouvelles perspectives pour les applications des méthodes électromagnétiques basse fréquence en archéologie

283 Rémy WASSONG \& Bruno GAVAZZI

Apport des prospections magnétiques haute résolution à la compréhension d'un habitat protohistorique :

l'exemple du site de hauteur fortifié du Maimont 


\title{
DYNAMIQUE GÉOMORPHOLOGIQUE HOLOCÈNE ET OCCUPATION HUMAINE DANS LE BASSIN-VERSANT DE L'EURE (BASSIN DE PARIS, FRANCE) : POTENTIELS D'UNE APPROCHE GÉOARCHÉOLOGIQUE MULTISCALAIRE ET DIACHRONIQUE
}

\author{
Théophile PIAU 1, 2, *, François BÉTARD 1, Fabienne DUGAST ${ }^{2}$, \\ Gilles ARNAUD-FASSETTA ${ }^{1}$, Vincent VIEL ${ }^{1}$ \\ ${ }^{1}$ Université de Paris, UMR 8586 PRODIG \\ 2 UMR 8167 Orient \& Méditerranée, CNRS \\ *theophilepiau@yahoo.fr
}

\section{RÉSUMÉ}

Localisé dans la partie occidentale du Bassin parisien, le bassin-versant de l'Eure présente une occupation humaine plurimillénaire. À l'interface entre plusieurs groupes culturels dès le Néolithique (groupes du Gord, de I'Artenac et de Bretagne), la vallée de I'Eure est un espace archéologique clef qui offre un cadre de recherche privilégié pour mettre en œuvre une approche géoarchéologique multiscalaire et diachronique combinant : (1) cartographie géomorphologique, comme outil permettant d'appréhender les biais taphonomiques dans la préservation des gisements archéologiques, (2) prospections géophysiques (tomographie de résistivité électrique) pour une reconstitution 2D de l'architecture du remblaiement alluvial, (3) acquisition et traitement des données stratigraphiques par carottage et analyses sédimentologiques. L'apport des archives sédimentaires fluviatiles est également mis en avant afin de reconstituer la trajectoire hydro-sédimentaire de I'Eure durant l'Holocène.
Located in the western part of the Paris Basin, the Eure catchment is marked by a several thousand years old human occupation. At the interface between several cultural groups since the Neolithic period (Gord, Artenac and Brittany), the Eure valley is a key archeological area for performing geoarchaeological investigations. The aim of this multiscale approach is to combine (i) geomorphological mapping in order to underline the geomorphological dynamics impacts on the taphonomy of settlement and archeological remains in the middle Eure valley; (ii) geophysical surveys (Electrical Resistance Tomography) to obtain a 2D view of the sediment structures of palaeochannels; and (iii) percussion drilling of sedimentary cores and sedimentological analyses. In addition, the sedimentary records of the Eure valley are a crucial indicator to reconstruct the hydro-sedimentary dynamics of the Eure catchment and the relationships between societies and their environment during the Late Holocene.
KEYWORDS

Geoarchaeoloy, paleoenvironment Holocene, Eure Valley, Paris Basin vallée de l'Eure, Bassin de Paris. 
À I'heure où la notion « d'Anthropocène » occupe le devant de la scène scientifique tout en étant sujette à de nombreuses controverses [1], l'étude des interactions entre les sociétés anciennes (du Néolithique au Moyen Âge), les écosystèmes et les oscillations climatiques à l'Holocène s'avère fondamentale afin de mieux comprendre les réponses des environnements contemporains face aux changements globaux et proposer des perspectives de gestion adaptées [2]. L'intérêt des systèmes géomorphologiques réside dans le fait qu'ils enregistrent avec une résolution temporelle parfois très fine (temps d'une crue) des archives sédimentaires et paléoécologiques (pollens, mollusques) qui permettent de retracer I'histoire des paysages [3]. Cependant, démêler la part respective des forçages naturels et de l'action anthropique dans les métamorphoses de ce continuum, des plateaux et vallons élémentaires aux plaines alluviales, est complexe et nécessite d'adopter une démarche pluridisciplinaire (archéologie, géomorphologie, géosciences), multiscalaire (de l'échelle régionale du bassin-versant à l'échelle locale du site archéologique) et diachronique (du temps long géologique au temps court de l'événement historique et hydrologique). Les recherches géoarchéologiques et paléoenvironnementales multi-proxies (indicateurs géomorphologiques et bio-indicateurs) conduites le long de transects représentatifs (intra- et hors sites archéologiques) sur la Seine et ses principaux affluents (Marne, Oise, Yonne) ont permis de proposer un premier modèle de la trajectoire hydro-sédimentaire des organismes fluviaux du Bassin parisien face aux perturbations bio-climatoanthropiques des dix derniers millénaires [4]. Les synthèses récentes soulignent la variabilité spatio-temporelle des réponses environnementales face aux forçages anthropo-climatiques, reflet de I'hétérogénéité de l'espace, des « effets de site » et de facteurs morphologiques d'échelle plus locale (cadre morpho-structural, système de pentes) [5].

Localisé dans la partie occidentale du Bassin parisien, le bassin-versant de l'Eure offre un cadre de recherche privilégié pour appréhender les mécanismes d'interaction sociétés-milieux au cours de l'Holocène à différentes échelles spatiales (régionale à locale) et temporelles (millénaire à annuelle). Trait d'union entre la Seine au nord et la Loire au sud (fig. 1A et B), la vallée de l'Eure se développe à l'interface entre plusieurs groupes culturels dès le Néolithique (groupes du Gord, de I'Artenanc et de Bretagne). Elle est marquée, durant la période antique, par l'émergence des Aulerques Éburovices au nord, des Carnutes au sud et des Durocasses à I'ouest, puis par les différents héritages politiques à l'époque mérovingienne et les conflits entre le duché de Normandie et le royaume de France au cours du Moyen Âge (fig. 2A et B). Elle apparaît de ce fait comme un espace archéologique clef pour appréhender les dynamiques du réseau de peuplement humain et la formation des cultures matérielles aux influences multiples sur la longue durée. Paradoxalement, elle s'inscrit dans une portion de territoire où la documentation archéologique et géomorphologique est hétérogène et peu exploitée.

L'article se propose d'établir un premier bilan d'une recherche exploratoire engagée en 2019 dans le cadre d'un mémoire de Master 2 puis d'une thèse de doctorat (2019-2022, directeur : François Bétard) au sein de I'Université de Paris (ED 624) et de I'UMR 8586 PRODIG. Adossée au programme collectif de recherche "Vallée de I'Eure : une rivière, des territoires » (coordination : Fabienne Dugast, financement principal : DRAC Normandie) et menée conjointement aux prospections archéologiques, l'analyse géomorphologique a eu pour ambition d'évaluer les potentialités paléoenvironnementales des archives sédimentaires de l'Eure moyenne dans une perspective géoarchéologique. Le choix de ce terrain d'étude s'est imposé pour plusieurs raisons. À la différence de la confluence Eure-Seine au nord du bassin-versant (boucle de Vaudreuil. fig. 1B) ou de I'agglomération chartraine au sud, la moyenne vallée de l'Eure est une portion de territoire qui demeure mal connue. En effet, ce secteur se singularise par un maigre corpus de données archéologiques qui pose la question de la conservation et/ ou de la visibilité des gisements dans le paysage et

[1] MAGNY 2019.

[2] BRAVARD \& MAGNY 2002.

[3] BRown 1997 ; LeSPez 2012 ; Berger ET AL. 2018.

[4] PAstre et al. 1997, 2006.

[5] LeSPeZ 2012 ; Gonet 2018 ; BeAuchamp 2019. 

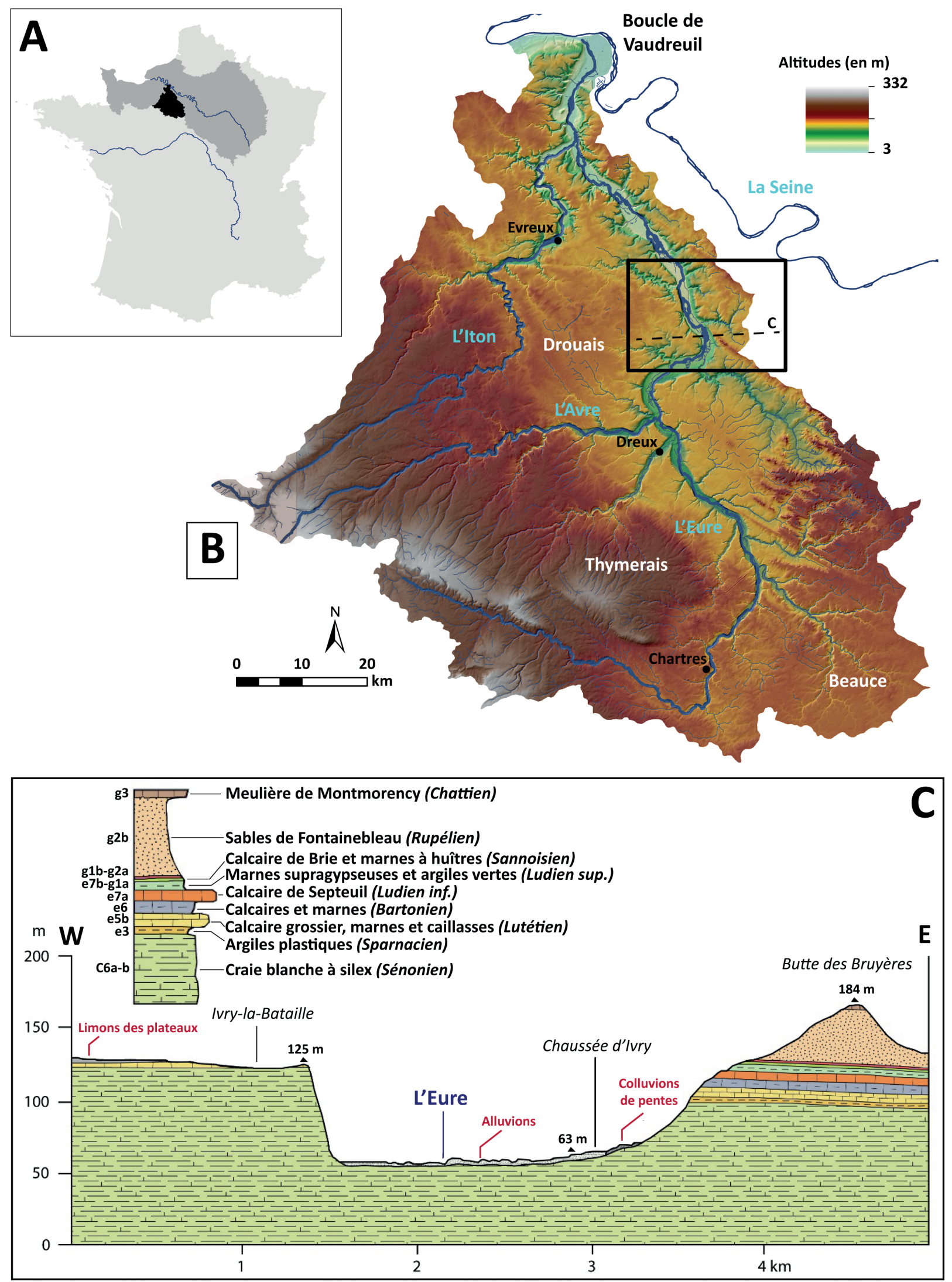

\section{Figure 1}

Carte de localisation de la zone d'étude $(A)$ et délimitation du bassin-versant de l'Eure (B). Le rectangle noir localise la fenêtre d'étude retenue pour ce travail exploratoire. C. Coupe géologique W-E à travers la vallée de l'Eure à la Chausséed'Ivry : une vue d'ensemble de la stratigraphie du bassin-versant. DAO : T. Piau (d'après BÉTARD ET AL. 2020). Fonds orographique : BD Alti $25 \mathrm{~m}$ de l'IGN, Fonds hydrographique : BD Carthage de l'IGN, Système de coordonnées : Lambert 93 Conformal Conic. 

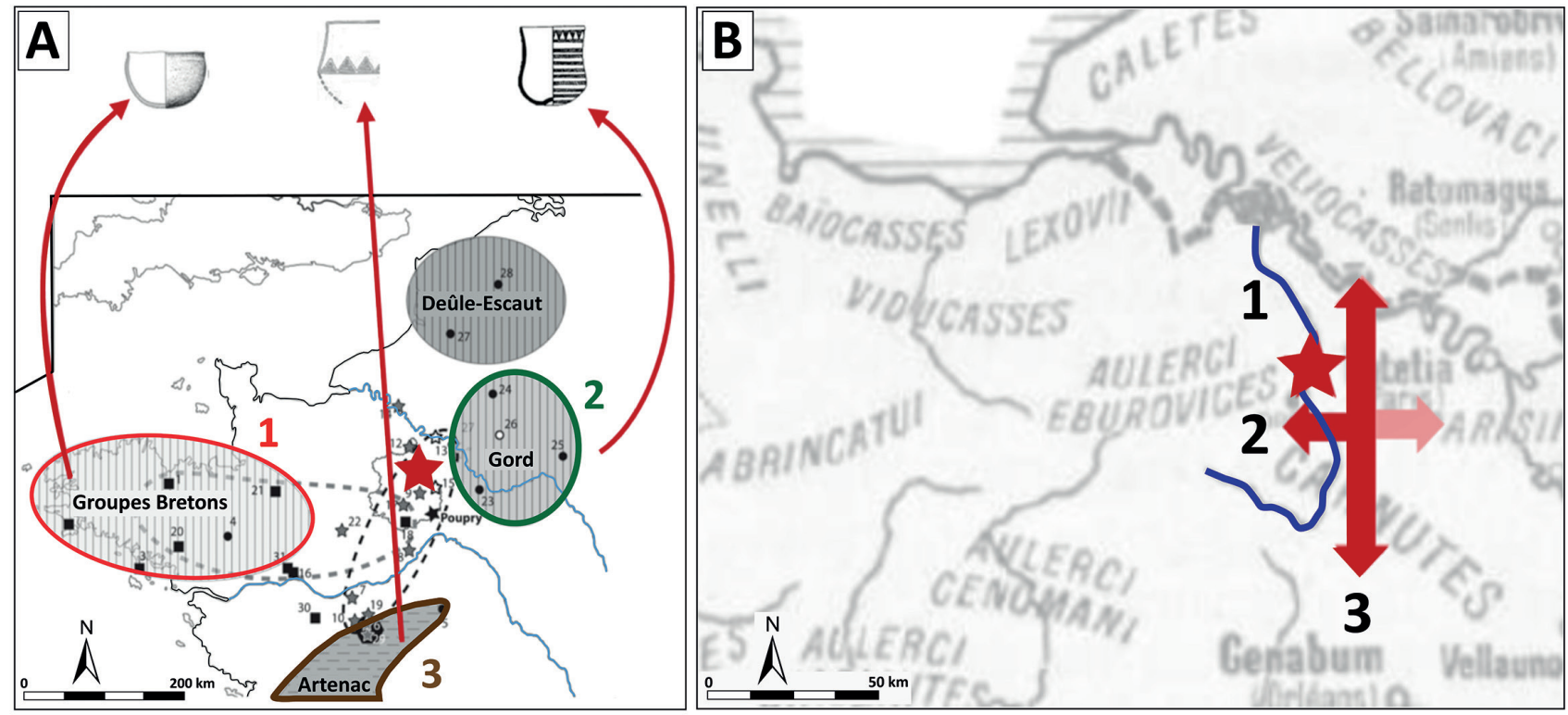

Figure 2

Groupes culturels du Bassin parisien. Sur les deux cartes, la moyenne vallée du bassin-versant de l'Eure est symbolisée par l'étoile rouge. Noter sa position d'interface durant le Néolithique (A) entre les groupes de Bretagne à l'ouest (1), de Gord au nord-est (2) et de l'Artenac au sud (3) puis, durant l'Antiquité (B) entre la cité des Aulerques-Eburovices au nord (1), des Carnutes au sud (3) et des Durocasses à l'ouest. DAO : T. Piau (d'après DugAST 2019).

des processus géomorphologiques à l'origine de leur fossilisation (alluvionnement, colluvionnement) ou de leur démantèlement (érosion), en lien avec la taphonomie des sites. Le cadre morpho-structural du bassin-versant de l'Eure est par ailleurs sensiblement différent entre la rive gauche, où la surface des plateaux est sous-tendue par les couches sédimentaires du Secondaire, et la rive droite, où ils sont principalement soulignés par les dépôts variés du Tertiaire (fig. 1C) [6]. Ainsi, les sous-bassins de rive droite de l'Eure pourraient avoir subi des évolutions asynchrones et/ou des réponses morpho-sédimentaires différentes des sous-bassins à lithologie crayeuse de rive gauche. Cette configuration géologique amène donc à s'interroger sur le rôle respectif de chacun des forçages (anthropiques et climatiques) et des effets des emboîtements d'échelle sur la propagation des signaux sédimentaires, des vallons élémentaires au fond de vallée de I'Eure, en lien avec l'organisation spatiale du peuplement humain. L'article synthétise les résultats préliminaires de cette recherche qui s'est articulée autour d'un double objectif : (1) à une échelle régionale, quel est l'impact des processus géomorphologiques dans la préservation des gisements archéologiques ? (2) à une échelle locale, quelles

[6] BÉTARD ET AL. 2020. sont les potentialités paléoenvironnementales des séquences sédimentaires préservées dans le fond de vallée de l'Eure à proximité des sites archéologiques identifiés ?

\section{LA MOYENNE VALLÉE DE L'EURE, CADRE DE RECHERCHE PERTINENT POUR UNE APPROCHE GÉOARCHÉOLOGIQUE ET PALÉOENVIRONNEMENTALE}

La fenêtre d'étude est située dans la section médiane du bassin-versant de l'Eure, entre les communes de Marcilly-sur-Eure au sud-ouest et Gadencourt au nord-ouest, sur un tronçon d'une trentaine de kilomètres. Le fond de vallée, d'une largeur de $1,5 \mathrm{~km}$, à fond plat et aux versants dissymétriques, s'encaisse de $70 \mathrm{~m}$ à partir de la surface de référence représentée par des plateaux culminant entre 170 et $130 \mathrm{~m}$. L'Eure a entaillé, en rive gauche, les assises crayeuses du Sénonien (Ivry-laBataille) et a épargné sur son versant de rive droite une grande partie des dépôts tertiaires qui s'échelonnent de I'Éocène au Mio-Pliocène (Chausséed'Ivry. Fig. 1C). La surface des plateaux est nappée de manteaux d'altérites (formations résiduelles à silex), de biefs à silex (en rive gauche) et de limons éoliens. Le long de la vallée, plusieurs niveaux de terrasses, étagées et partiellement masquées par 


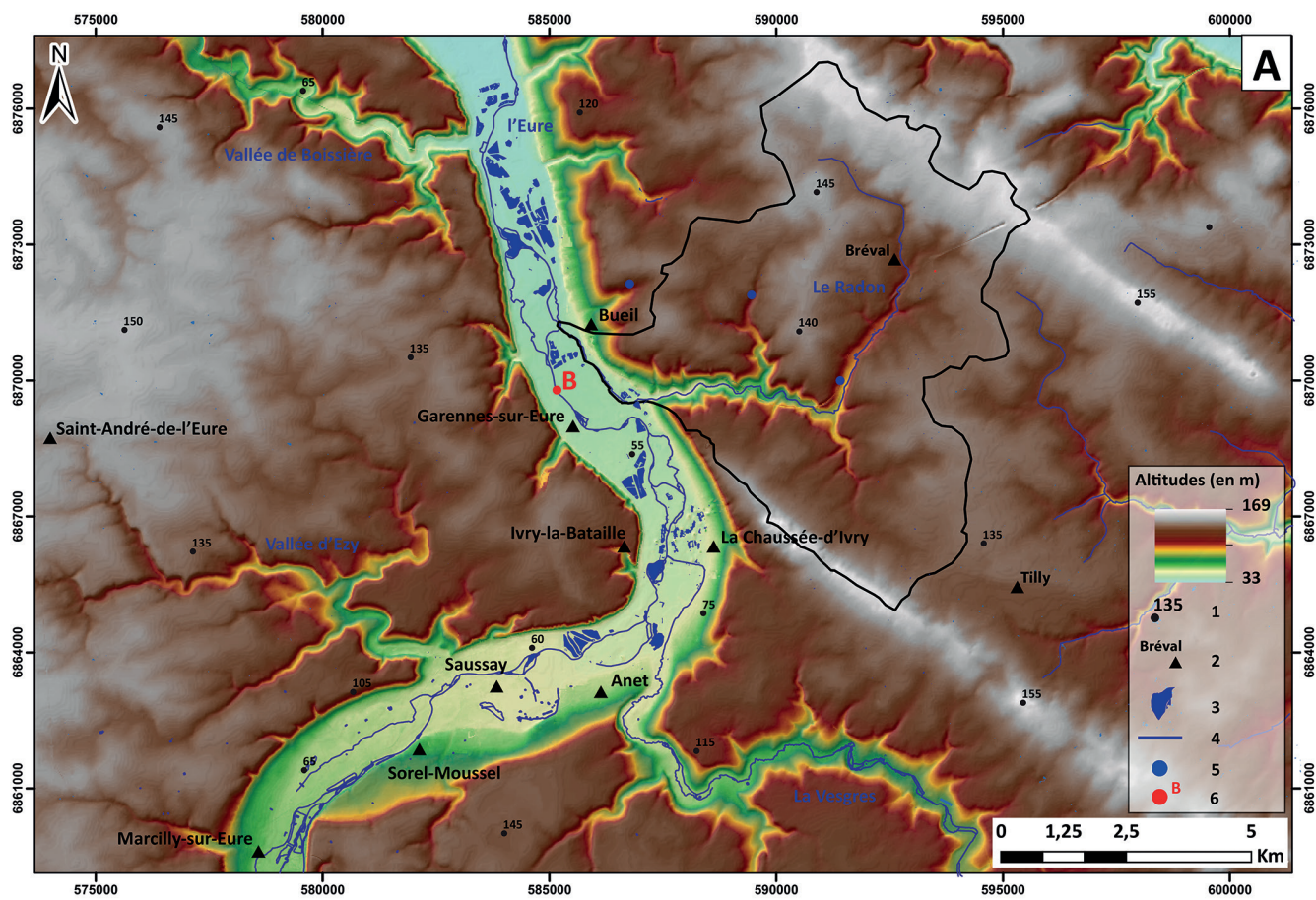

B

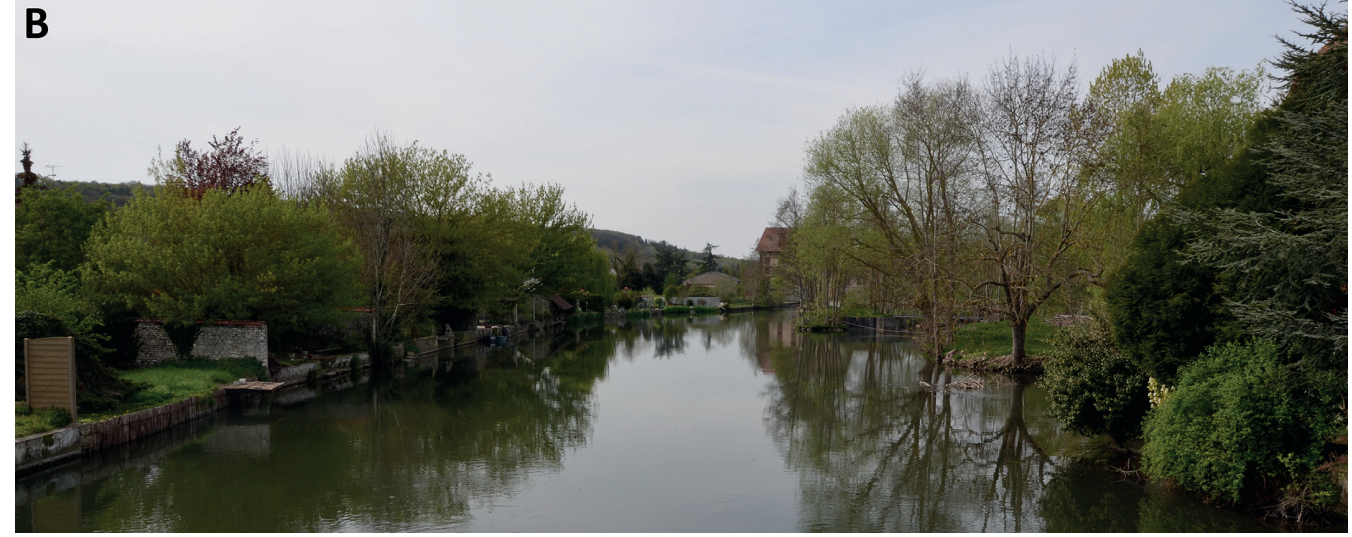

Figure 3.

A. Contexte topographique et hydrographique de la fenêtre d'étude, centrée sur la moyenne vallée de l'Eure.

Légende :

1. point coté,

2. commune,

3. ballastière,

4. cours d'eau,

5. exsurgence karstique,

6. localisation de la photographie B.

B. L'Eure à Garennes-sur-Eure, ici un tronçon largement aménagé et rectifié.

DAO et Photo

T. Piau. Fonds orographique : BD Alti $25 \mathrm{~m}$ de l'IGN, Fonds hydrographique : BD Carthage de I'IGN, Système de coordonnées : Lambert 93 Conformal Conic. d'importants dépôts colluviaux, sont individualisés entre Anet et Bueil. Le fond de vallée est tapissé d'alluvions fluviatiles holocènes surmontant la grave grossière weichsélienne. L'Eure coule depuis Nogentle-Roi selon une orientation SSE/NNW avant de s'infléchir de près de $90^{\circ}$ au niveau de Sorel-Moussel, puis de reprendre son orientation originelle à partir d'Ivry-la-Bataille [7]. Ce tracé en baïonnette serait lié à I'intersection entre I'Anomalie Magnétique du Bassin de Paris et l'accident linéamentaire « AvreEure-Seine-Oise »[8]. Sur le tronçon étudié, le cours de l'Eure est artificialisé par divers aménagements et activités anthropiques modernes (berges partiellement maçonnées, ballastières) et se caractérise par un chenal unique méandriforme (fig. 3 A et B). En rive droite, ses deux principaux affluents sont la Vesgres et le Radon, tandis qu'en rive gauche, deux vallées sèches d'orientation NW-SE (vallée d'Ezy et de la Boissières) viennent se raccorder au fond de vallée. Ces dernières ont la particularité d'avoir un cours supérieur relativement rectiligne qui, dans sa partie aval, évolue en une série de méandres peu encaissés dans la craie [9]. La nature plus ou moins perméable des couches géologiques affleurantes permet d'expliquer les différences de drainage entre la rive gauche, où le chevelu hydrographique est quasi inexistant sur les couches crayeuses, et la rive droite, où il est beaucoup plus développé du fait de

[7] D'après les données de la Banque Hydro, le module de l'Eure, près de l'exutoire à Louviers, est de $26,2 \mathrm{~m}^{3} / \mathrm{s}$ pour une puissance spécifique moyenne de $2 \mathrm{~W} / \mathrm{m}^{2}$, un trait typique des rivières de basse énergie et à lente capacité de réajustement morphodynamique dans BROOKES 1988.

[8] Obert et al. 1992 ; 1997 ; Lacombe \& Obert 2000.

[9] DeWOLF 1974. 


\begin{tabular}{|c|c|c|}
\hline Catégorie de données & Nature de la donnée & Source de la donnée \\
\hline Topographiques & $\begin{array}{c}\text { MNT BD ALTI } 25 \mathrm{~m} \\
\text { MNT RGE ALTI } 5 \mathrm{~m} \\
\text { MNT RGE ALTI } 1 \mathrm{~m}\end{array}$ & IGN / Géoportail \\
\hline $\begin{array}{c}\text { Géologiques et } \\
\text { géomorphologiques }\end{array}$ & $\begin{array}{c}\text { Cartes à } 1 / 50000 \\
\text { BSS (logs géologiques) }\end{array}$ & $\begin{array}{c}\text { BRGM / Infoterre } \\
\text { CNRS / RCP 77 }\end{array}$ \\
\hline $\begin{array}{c}\text { Historiques et } \\
\text { archéologiques }\end{array}$ & $\begin{array}{c}\text { Inventaire des sites } \\
\text { archéologiques } \\
\text { Cartes anciennes et archives } \\
\text { historiques }\end{array}$ & $\begin{array}{c}\text { Archives départementales (Eure, } \\
\text { Eure-et-Loir, Yvelines) } \\
\text { INRAP }\end{array}$ \\
\hline
\end{tabular}

Figure 4

Synthèse des données utilisées pour la réalisation de la cartographie géomorphologique de la moyenne vallée de l'Eure. DAO. T. Piau.

la présence des niveaux imperméables du Tertiaire (fig. 1C). Plusieurs exsurgences sont d'ailleurs identifiées au contact entre le calcaire lutétien et les argiles/marnes imperméables dans le sous-bassin du Radon (fig. 3A).

Sur le plan archéologique, l'espace apparaît occupé de manière très contrastée, avec des zones remarquables non pas liées à l'occupation effective mais à une documentation archéologique fragmentaire. L'intensité de la recherche dans le fond de vallée et les plateaux adjacents reste très inégale. Une forte concentration de gisements archéologiques est observée dans les environs immédiats des grands centres urbains (Chartres et Dreux) et à la confluence Eure-Seine (boucle de Vaudreuil) touchés par de nombreuses opérations archéologiques préventives. A contrario, dans la moyenne vallée de l'Eure, le corpus de données archéologiques restreint ne permet pas d'analyser la complexité du réseau de peuplement et ses dynamiques et amène à s'interroger sur l'impact des processus géomorphologiques sur les conditions d'évolution et de conservation des vestiges archéologiques au cours du temps.

\section{MÉTHODOLOGIE : LE CHOIX D'UNE APPROCHE GÉOARCHÉOLOGIQUE MULTISCALAIRE ET DIACHRONIQUE}

Entre mars et avril 2019, les missions de terrain ont été consacrées à la réalisation d'une cartographie géomorphologique afin de proposer un zonage des potentialités paléoenvironnementales et géoarchéologiques d'un tronçon de la moyenne vallée du bassin versant de l'Eure. Ce volet s'est articulé autour de quatre principaux objectifs : (1) identifier les secteurs favorables à l'archivage de données sédimentaires à haute résolution temporelle et les processus géomorphologiques à l'origine de leur mise en place ; (2) évaluer la représentativité spatiale des séquences sédimentaires préservées dans le système géomorphologique ; (3) proposer une comparaison entre la répartition des gisements archéologiques et les formations superficielles quaternaires (argile à silex ; lœss ; colluvions ; alluvions) et (4) guider les prospections thématiques en cours et à venir. Principalement appuyée sur l'examen de la documentation disponible (fig. 4), I'analyse géomorphologique a été ponctuellement complétée par l'observation d'affleurements, de coupes naturelles (Guainville) et de sondages à la tarière pédologique dans le fond de vallée (site de Bellevue). La confrontation de ces données complémentaires, après leur géoréférencement et vectorisation dans un SIG (ArcMap 10.4), a contribué à spatialiser les formes et formations quaternaires (nappes et terrasses alluviales, glacis colluviaux et dépôts de pente, modelés et dépôts éoliens). Une zone atelier à fort potentiel géoarchéologique et paléoenvironnemental a par la suite été sélectionnée dans le fond de vallée de l'Eure, autour d'un sanctuaire d'époque romaine reconnu par photographie aérienne au lieu-dit Bellevue (Garennessur-Eure). L'analyse d'un modèle numérique de terrain à haute résolution (RGE Alti $1 \mathrm{~m}$ ) a permis d'identifier les traces probables de paléochenaux à proximité immédiate du complexe cultuel (fig. 5) [10]. Cet axe de recherche d'échelle plus locale avait I'ambition d'appréhender l'architecture

[10] BÉTARD ET AL. 2020. 


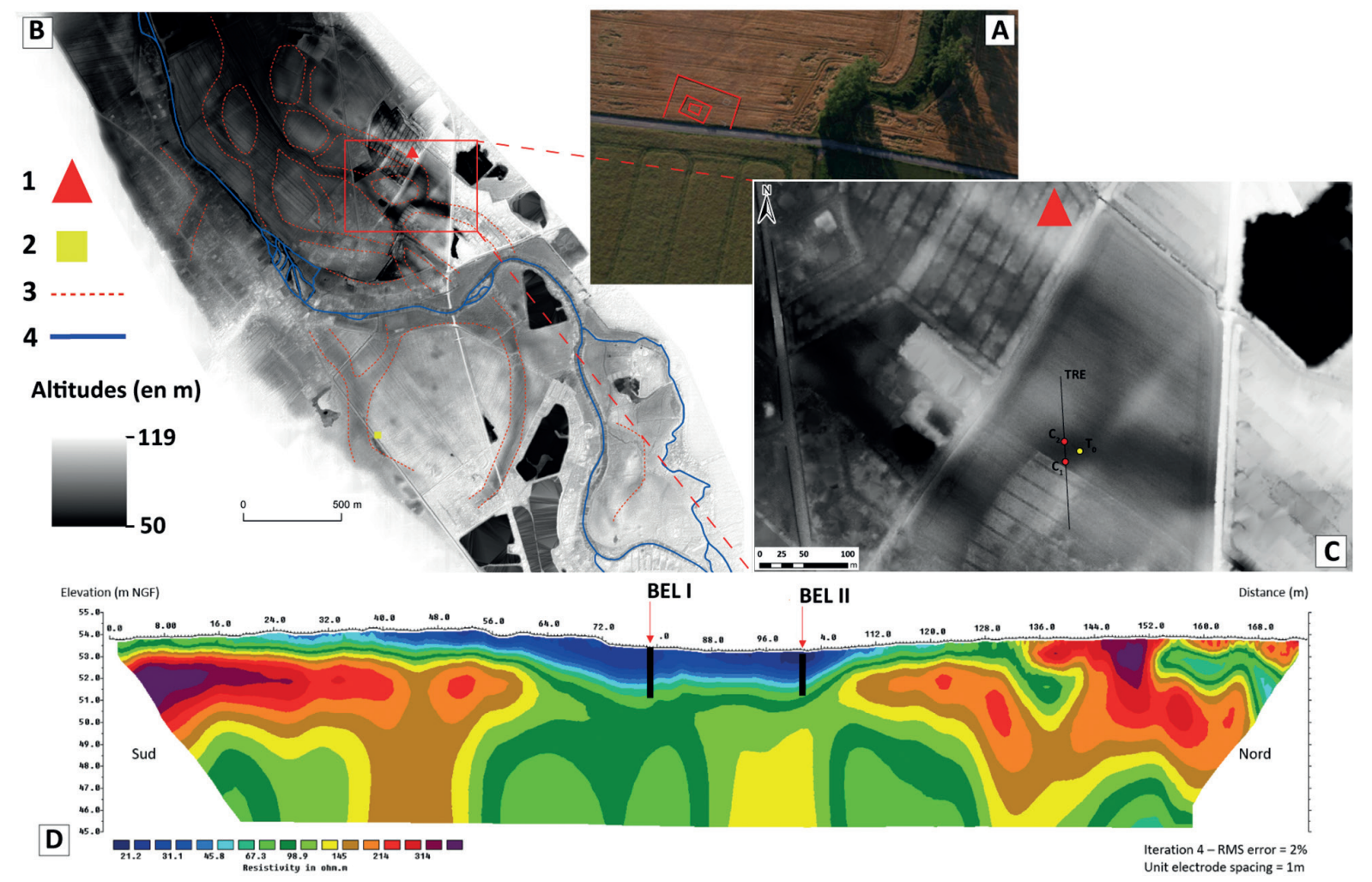

Figure 5

A. Sanctuaire antique de Bellevue repéré en prospection aérienne en 2011 par l'association Archéo27. B. Extrait du modèle numérique de terrain RGE Alti $1 \mathrm{~m}$ de l'IGN et interprétation géomorphologique et paléohydrographique préliminaire à proximité du sanctuaire gallo-romain de Bellevue (triangle rouge) sur la commune de Garennes-sur-Eure. Légende: 1. Fanum, 2. Voie antique, 3. Paléochenaux, 4. Chenaux actuels de l'Eure, C. Tracé du profil de résistivité électrique (TRE) perpendiculaire à l'axe d'un paléochenal de l'Eure et localisation des deux carottages. D. Sur le profil de résistivité électrique, les mesures de résistivité apparente forte en rouge renvoient à des faciès grossiers (grave à silex roulés). A contrario, les mesures de résistivité apparente faible (en bleu et vert) renvoient à des faciès sédimentaires plus fins (argiles et limons). DAO : T. Piau (d'après PIAU 2019). Fonds orographique : RGE Alti $1 \mathrm{~m}$ de I'IGN, Fonds hydrographique : BD Carthage de l'IGN, Fonds Archéologique : BD Archéo du PCR ValEuRT, Système de coordonnées : Lambert 93 Conformal Conic.

du remblaiement sédimentaire de la plaine alluviale de I'Eure afin de restituer le paléoenvironnement fluvial au moment de l'implantation du site antique. En complément de l'interprétation paléohydrographique, une campagne de prospection géophysique (tomographie de résistivité électrique) a été réalisée en mai 2019 à l'aide d'un système multi-électrodes Abem Terrameter LS, en collaboration avec Clément Virmoux, (UMR 8591 LGP). Afin de reconstituer la géométrie 2D des structures sédimentaires, le profil de Tomographie de Résistivité Électrique (TRE) a été positionné perpendiculairement à l'axe d'écoulement du paléochenal. Pour conserver une résolution suffisante, l'espacement entre chaque électrode a été de $1 \mathrm{~m}$ pour une profondeur d'investigation d'environ $11 \mathrm{~m}$ (dispositif de «Schlumberger»). Début juin 2019, deux carottages (carottier à percussion cobra MK1) ont été implantés dans le remblaiement du paléochenal afin de préciser l'architecture et l'âge des unités sédimentaires préalablement individualisées sur le profil TRE. Des analyses sédimentologiques en laboratoire (granulométrie, paramètres texturaux et image $\mathrm{CM}$ ) sont venues étayer les observations texturales et structurales des unités, contribuer à caractériser les moments et les milieux de sédimentation fluviatile associés, et finalement préciser les conditions paléohydrologiques. Une datation radiocarbone $\left({ }^{14} \mathrm{C}\right.$ Accelerated Mass Spectrometry) a été exécutée au laboratoire BetaAnalytic sur un échantilIon de sédiment organique prélevé sur la carotte BEL II. La date obtenue a été calibrée à 2 sigma avec le logiciel OxCal 4.3 (IntelCal13) [11].

[11] Reimer ET AL. 2013. 


\section{RÉSULTATS ET PREMIÈRES INTERPRÉTATIONS}

\section{CARACTÉRISTIQUES \\ GÉOMORPHOLOGIQUES DE LA \\ MOYENNE VALLÉE DE L'EURE}

\section{Des surfaces substructurales dominées par quelques buttes-témoins et entaillées par des vallées dissymétriques}

Au sein de la moyenne vallée de l'Eure, les principaux reliefs structuraux sont les surfaces substructurales éocènes des plateaux (calcaire « grossier 》 d'âge lutétien, calcaire de Septeuil d'âge ludien), parfois étagées (avec des contrastes de lithologie en rive droite), où se détachent quelques buttes-témoins oligocènes armées de meulière (Butte des Bruyères) et dans lesquelles s'inscrit le réseau hydrographique actuel. Même si la tectonique (synclinal de l'Eure, faille de la Seine) et les formes structurales commandent les grandes lignes du relief, ce dernier est largement modelé et remanié par les dynamiques fluviales, éoliennes et périglaciaires quaternaires (fig. 6 et 7A).

Les modelés de versant et de vallée sont liés à la lithologie, à l'occupation des sols mais aussi et surtout à l'action des écoulements superficiels (du ruissellement diffus du sommet des interfluves à l'écoulement concentré des vallons et des vallées principales). C'est le cas des versants raidis par sapement fluvial en rive gauche de l'Eure, à proximité de Bueil. Il est cependant délicat de distinguer les versants réguliers liés au sapement fluvial de ceux « réglés », c'est-à-dire façonnés par les dynamiques périglaciaires puisqu'ils présentent le même profil d'équilibre de I'ordre de 20 à $25^{\circ}$. Généralement, ces deux processus se combinent dans l'espace et dans le temps. Les processus périglaciaires sont à l'origine d'un remaniement plus ou moins profond et étendu des formations superficielles. La cryoturbation, les fentes de gel et coins de glace affectent la partie supérieure de la nappe alluviale de Guainville (fig. 7B). Les processus périglaciaires ont également contribué à façonner les profils des vallons (à fond plat, en berceau et en $\mathrm{V}$ ). La majorité des dissymétries des vallées et vallons rencontrés en rive gauche de l'Eure sur substrat crayeux est d'origine climatique. Les vallées sèches d'Ezy et de Boissière présentent une nette dissymétrie : les versants «froids » face à l'est sont en pente douce et recouverts de limons colluvionnés (versant d'accumulation par déflation, solifluxion et gélifluxion), alors que les versants « chauds», face à l'ouest, sont taillés dans la craie et sont plus courts et raides (versant d'érosion). Enfin, la troncature des sols lœssiques (sol brun lessivé) est accentuée par les pratiques agro-pastorales qui se développent dès le Néolithique [12]. Cette dernière est d'autant plus importante en rebord de plateau que la couverture lœssique est peu épaisse. Le substrat calcaire est d'ailleurs affleurant dans ce secteur.

\section{Des formes et formations quaternaires favorables à la conservation des archives paléoenvironnementales}

\section{Nappes et terrasses alluviales}

Les dépôts alluviaux d'âge quaternaire sont composés des alluvions de l'Eure et de ses affluents : en rive droite, La Vesgres à l'extrémité sud et le Radon plus au nord; en rive gauche, les vallées sèches d'Ezy au sud et de Boissières à l'extrémité nord de la carte (fig. 6). Les formations les plus anciennes sont disposées en terrasses étagées et occupent principalement le versant est de la vallée de l'Eure en rive droite. Elles forment des replats d'extension et de puissance variable et sont masquées localement par des dépôts colluviaux dans la moitié nord de la fenêtre d'étude, entre Bueil et Breuilpont. Une nappe alluviale (notée Fyd sur la carte géologique) d'une puissance de $4 \mathrm{~m}$ est individualisée en pied de versant à Guainville, à une altitude relative d'environ $+10 \mathrm{~m}$. Par analogie avec d'autres séquences alluviales décrites par Y. Dewolf [13] dans la vallée de l'Eure à la même altitude (Bueil, Chaudon), cette séquence est probablement d'âge saalien [14]. Elle est composée d'une alternance de sables fins à grossiers orangés plus ou moins limoneux ou argileux contenant des intercalations de lits subhorizontaux à galets de silex anguleux à sub-émoussés. Sur le terrain, des dépôts tufacés ont été identifiés dans le comblement d'un paléochenal dans la partie médiane de l'affleurement. La partie sommitale de la coupe est constituée d'un niveau à cailloutis de silex emballés dans une matrice argilo-sableuse ferruginisée (paléosol éemien ?) affectée par des figures de cryoturbation et des fentes de gel à rapporter au Weichsélien. Ce paléosol est recouvert de limons éoliens mêlés à des colluvions weichséliennes en provenance du versant (fig. 7B). Une campagne de

[12] WUSCHER ET AL. 2012.

[13] DeWOLF 1970.

[14] GuilLemin 2017. 


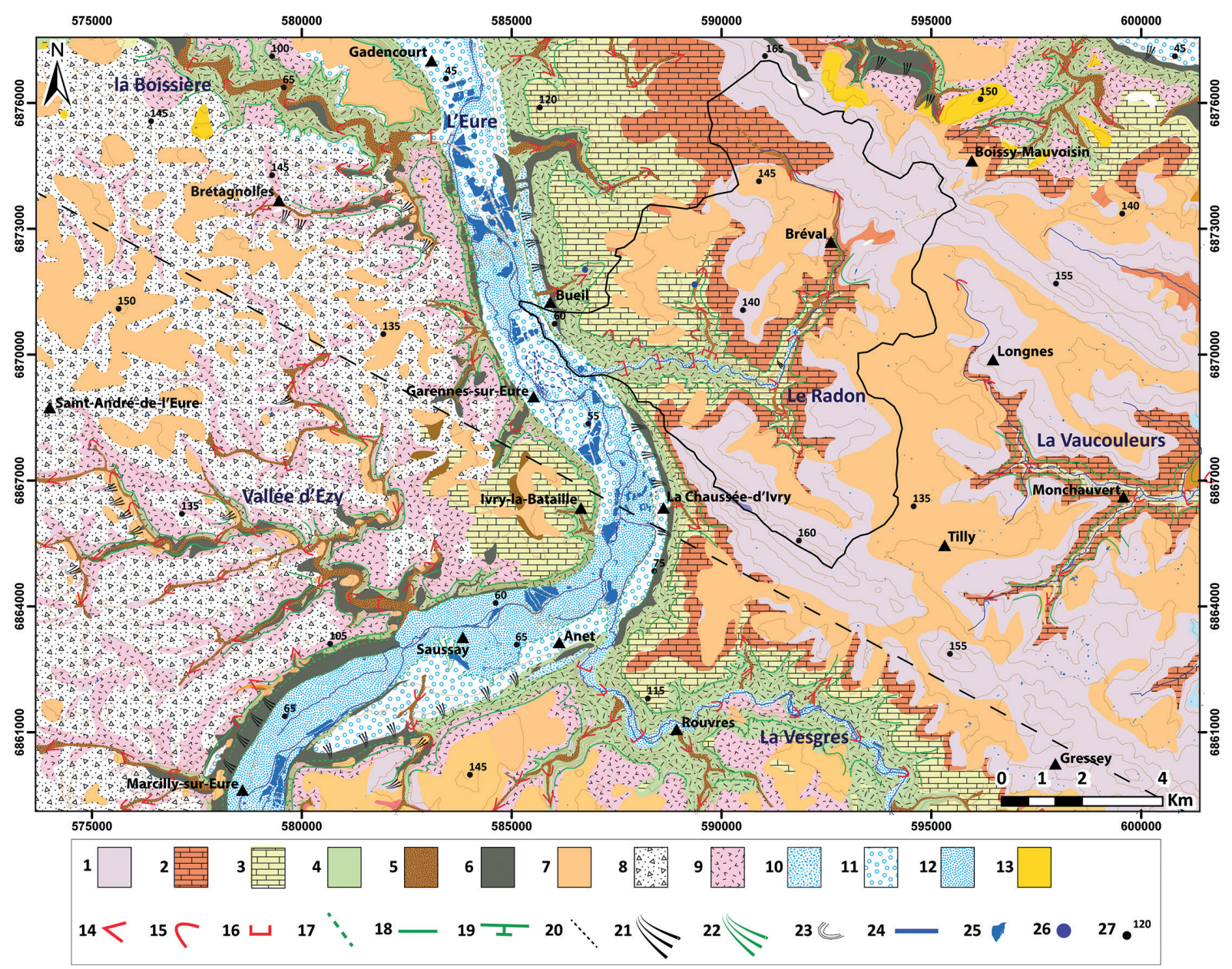

Figure 6

Carte géomorphologique de la moyenne vallée de l'Eure. Légende : 1. bancs meuliérisés encadrant une épaisse couche sableuse (g2, g1), Stampien-Sannoisien, 2. intercalations calcaires dans marnes et sables (e7, e6), Ludien-Bartonien, 3 calcaire grossier sur argiles et sables (e5, e3), Lutétien-Yprésien, 4. craie à silex (c6-c5), Campanien, 5. colluvions de fond de vallon et vallée, $\mathbf{6}$. colluvions de versant, 7. lœss, 8. bief à silex, 9. argile à silex, 10. alluvions de moyenne terrasse $(F x)$, 11. alluvions de basse terrasse $(F y)$, 12. alluvions récentes $(F z), 13$. sables de Lozère, 14. vallon en $V, 15$. vallon en berceau, 16. vallon à fond plat, 17. rupture de pente concave, 18. rupture de pente convexe, 19. versant régulier lié au sapement fluvial, 20. axe synclinal, 21. glacis colluvial et colluvio-alluvial, 22. glacis alluvial, 23. courbes de niveau, équidistance $10 \mathrm{~m}, 24$. chenal d'écoulement permanent, 25. étang ou ballastière inondée, 26. source, 27. cotes en mètres. DAO : T. Piau. Fonds orographique : BD Alti $25 \mathrm{~m}$ de l'IGN, Fonds hydrographique : BD Carthage de l'IGN, Fonds géologique : BD Charm-50 BRGM, Système de coordonnées : Lambert 93 Conformal Conic.

datation OSL (Optically Stimulated Luminescence) est envisagée en 2020 afin d'établir le cadre chronostratigraphique de cette séquence de référence. Plus en amont, entre les communes de Sorel-leMoussel et d'Anet, une succession de trois nappes alluviales étagées est individualisée sur environ $500 \mathrm{~m}$ de large pour $5 \mathrm{~km}$ de long à des altitudes relatives comprises entre $+5,+15$ et $+25 \mathrm{~m}$ [15]. Les alluvions holocènes tapissent le fond de vallée de l'Eure sur une épaisseur pouvant atteindre $10 \mathrm{~m}$ en pied de versant à la Chaussée-d'Ivry, d'après les logs de la Banque du Sous-Sol. Essentiellement limoneuses au sommet, elles présentent localement des niveaux plus argileux, sableux et parfois tour- beux. Les alluvions holocènes reposent sur la grave grossière weichséliennne mise en place à la fin du Pléniglaciaire supérieur et remaniée au cours du Tardiglaciaire. Cette grave à silex est affleurante, voire subaffleurante dans le fond de vallée sur certaines parcelles agricoles où elle s'apparente à un niveau de basse terrasse. Plusieurs paléoformes fluviales (paléochenaux et montilles sableuses) ont été décelées dans la topographie actuelle, en particulier à proximité du sanctuaire antique de Bellevue

[15] FENCKE ET AL. 2016. 


\section{A}

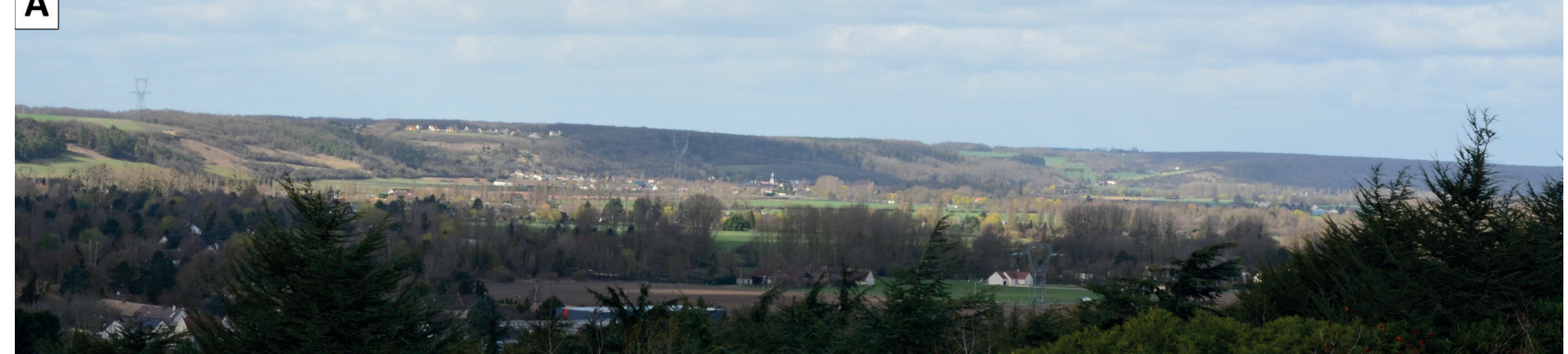

acis
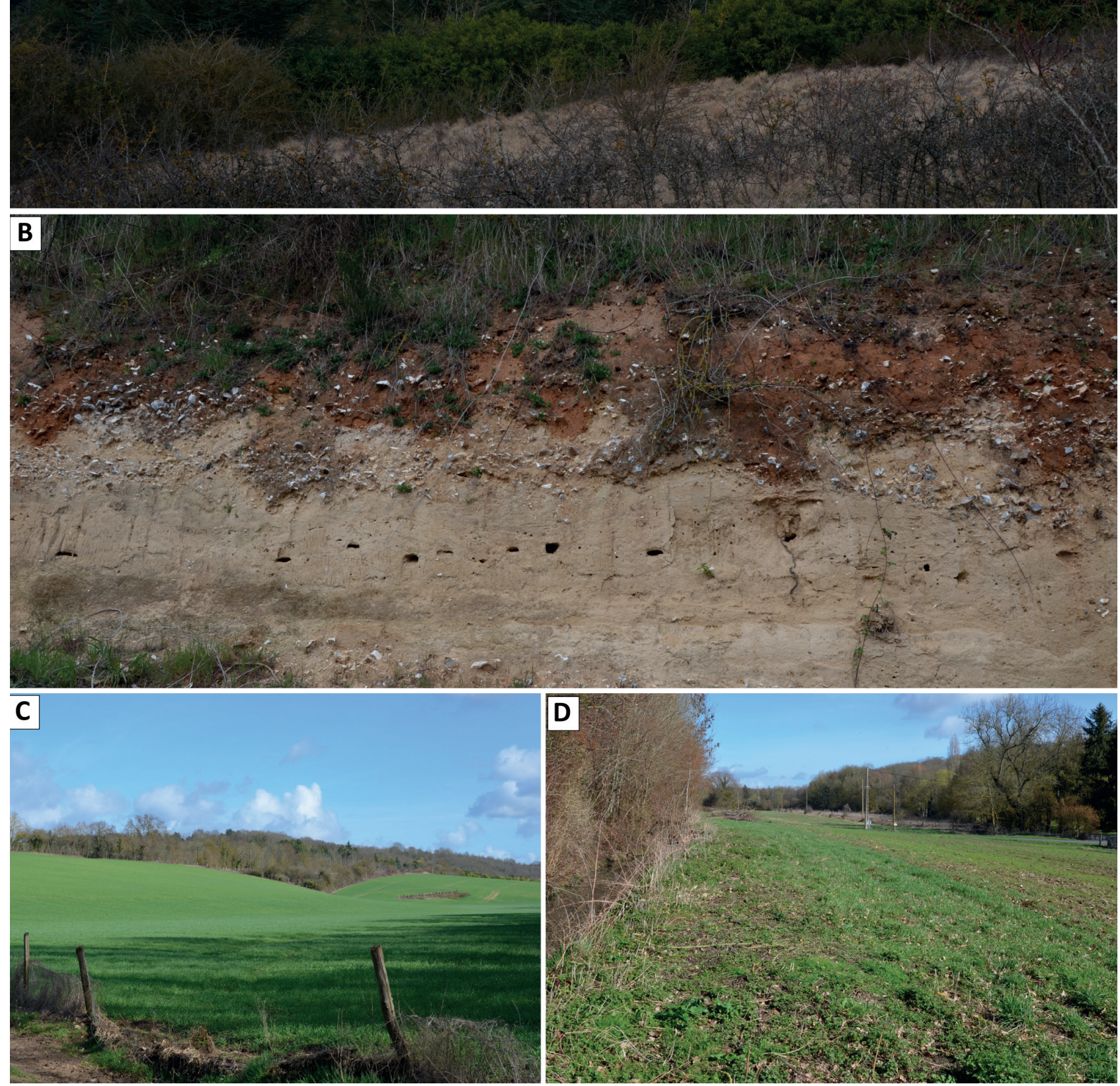

Figure 7

A. Vue en direction du nord-est de la vallée de l'Eure depuis le plateau en rive droite en aval de la Chaussée-d'Ivry. De part et d'autre de la vallée de l'Eure, la surface de ce plateau correspond pro parte à une surface substructurale éocène. Ce panorama permet d'observer, au second plan, le versant crayeux de la rive gauche de l'Eure réglé par le sapement fluvial et les processus périglaciaires quaternaires. B. Terrasse alluviale de Guainville. Noter, dans la partie sommitale de l'affleurement, les nombreuses marques de cryoturbation, fentes de gel et coins de glace remaniant le niveau sous-jacent ferruginisé. C. Basse vallée du Radon. Noter le faible remblaiement alluvial du fond de vallée, le versant se raccordant directement au fond de vallée. D. Glacis colluvial identifié dans la moyenne vallée du Radon. Noter également le fossé de drainage agricole à l'extrémité gauche de la photographie, interrompant le glacis colluvial. Photos : T. Piau et F. Bétard, 2019. 
sur la commune de Garennes-sur-Eure. Concernant les petits affluents de l'Eure en rive droite, ils se distinguent de leurs voisins développés dans la craie. Les vallées du Radon et de la Vesgres se caractérisent par un profil transversal régulier et ne présentent qu'un drain unique à l'opposé des vallées crayeuses en rive gauche, qui s'organisent en un ensemble de petits drains multiples dans leur partie amont [16]. L'une et l'autre sont a priori marquées par l'absence d'un étagement des formes alluviales (fig. 7C). Aujourd'hui, le Radon est incisé d'un mètre environ dans les alluvions limoneuses de sa plaine alluviale et n'emprunte plus, sur certains tronçons (basse vallée), les points bas du fond de vallée. Cette migration de la rivière hors de son talweg semble être liée à son artificialisation croissante depuis la période médiévale (moulins à eau et biefs associés). La confluence du Radon avec I'Eure est totalement artificielle et, d'après les planches du cadastre napoléonien de la commune de Garennessur-Eure, antérieure à 1830 .

\section{Glacis colluviaux et dépôts de pente}

En rive gauche de l'Eure, dans les fonds de vallée drainés par des ruisseaux à écoulement temporaire et les vallons secs, les remblaiements colluviaux semblent peu épais $(<2 \mathrm{~m}$ [17]) en raison de leur forte pente ( $>10 \%$ ). Il est plus difficile d'établir la puissance du remblaiement en tête de vallon en l'absence de sondages. Les formations de bas de versant sont quasi systématiques au sein de la fenêtre d'étude. Elles concernent les versants crayeux abrupts de la vallée de l'Eure et, dans une moindre mesure, ceux de ses affluents où les pentes sont plus faibles (sous-bassin du Radon). Ces colluvions remobilisent les formations superficielles des plateaux et des versants (limons éoliens, argiles et biefs à silex) et se raccordent au fond de vallée sous forme de glacis. D'âge weichsélien à holocène, elles se sont mises en place par le biais de processus gravitaires (cryoclastie), de ruissellements concentrés et diffus sur les versants [18]. Enfin, les formations de «biefs à silex », c'est-à-dire des argiles à silex remaniées par les processus périglaciaires, occupent deux positions topographiques en rive gauche : les biefs de plateau et les biefs de versant (fig. 6).

\section{Modelés et dépôts éoliens}

La surface des plateaux adjacents à la vallée de l'Eure et les versants « sous le vent » (orientés au nord et à l'est) sont nappés d'une couverture limoneuse, irrégulière et discontinue, d'épaisseur métrique, déposée par les processus éoliens durant les épisodes froids et arides du Pléistocène [19]. Les séquences lœssiques sont plus épaisses au centre des plateaux, à proximité de la Vesgres (entre 0,80 et $1 \mathrm{~m}$ ) et peuvent atteindre jusqu'à 2,50 $\mathrm{m}$ à Bû plus au sud [20]. En l'absence de prospection approfondie sur les plateaux et les versants, aucune différenciation n'a été faite entre les sables limoneux, les limons non-calcaires et les limons calcaires. La carte géomorphologique produite dans le cadre de ce travail reprend l'extension des limons de plateau (puissance supérieure ou égale à $3 \mathrm{~m}$ ) des cartes géologiques au 1/50 000 (BdCharm-50).

\section{IMPACTS DES PROCESSUS GÉOMORPHOLOGIQUES QUATERNAIRES DANS LA PRÉSERVATION DES GISEMENTS ARCHÉOLOGIQUES : PREMIERS ÉLÉMENTS DE RÉPONSE}

La spatialisation des formes d'érosion et des formations d'accumulation permet d'apporter des éléments de réponse sur le rôle d'un éventuel biais taphonomique dans la distribution actuelle des gisements archéologiques (fig. 8). Les gisements identifiés dans le fond de vallée sont prioritairement implantés sur des terrasses alluviales surplombant de quelques mètres la plaine d'inondation de I'Eure. C'est le cas des enclos protohistoriques repérés en prospection aérienne dans la portion sud-est de la zone d'étude, entre Saussay et Anet. Ce replat est propice à l'implantation humaine car (1) suffisamment près de la ressource en eau pour pouvoir en bénéficier, (2) topographiquement isolé en cas d'épisodes de crue ( $5 \mathrm{~m}$ de dénivelé environ), (3) à la confluence entre les vallées de la Vesgres et de I'Eure. À l'échelle de I'Holocène, les terrasses alluviales anciennes sont fossilisées sous d'épais dépôts colluviaux du fait d'un important colluvionnement au pied des versants crayeux (glacis colluviaux entre Bueil et Breuilpont). En rive concave, I'absence de nappe alluviale bien développée et la présence de versants abrupts, réglés par les processus périglaciaires et le sapement fluvial, ont pu entraîner un démantèlement des gisements

[16] DEWOLF 1974.

[17] Ibid.

[18] Borderie \& ChAMAUX 2016.

[19] WUSCHER ET AL. 2012 ; Borderie 2014 ; BORDERIE \& CHAMAUX 2016.

[20] DEWOLF 1974. 


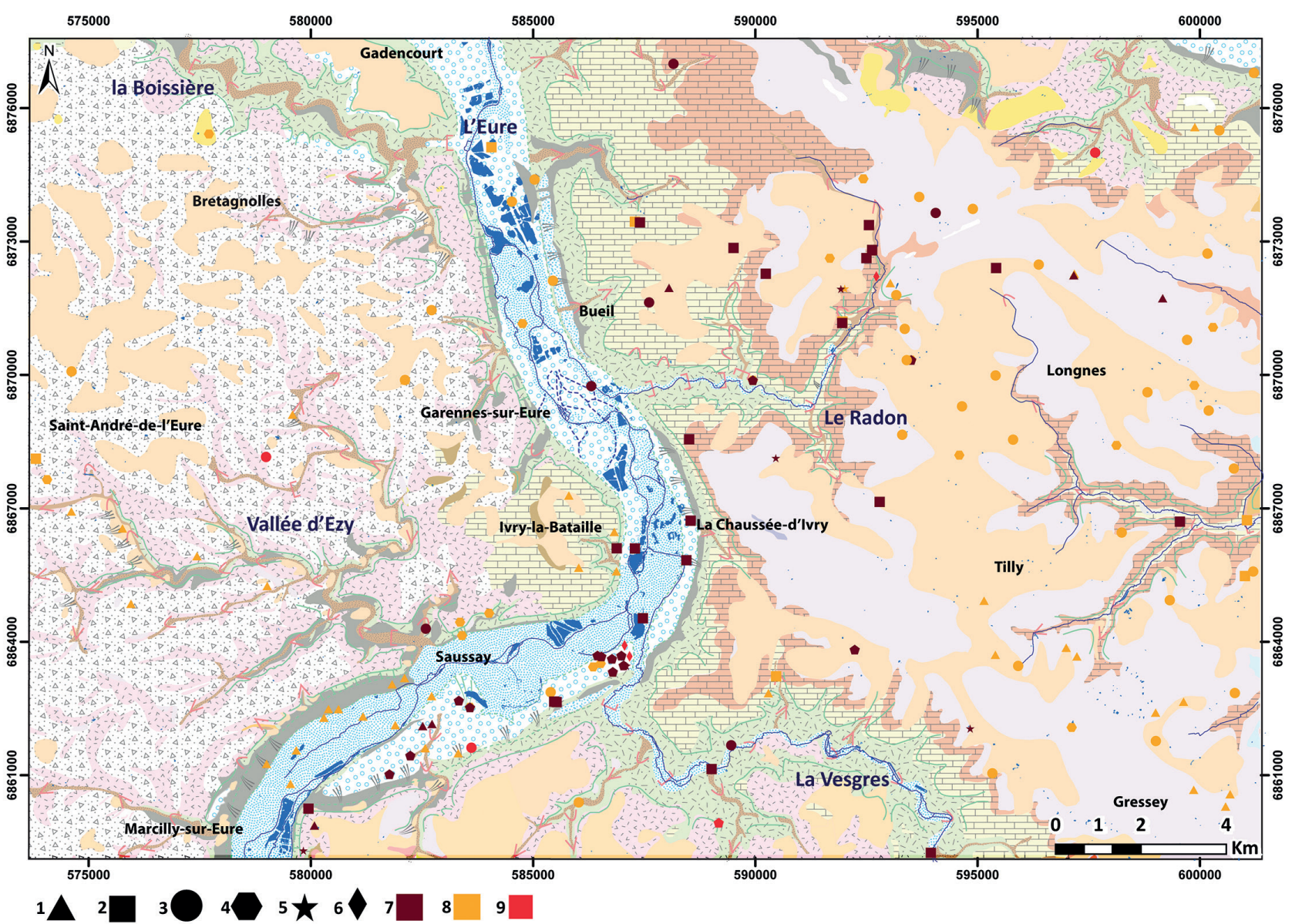

\section{Figure 8}

Comparaison entre la localisation des gisements archéologiques et celle des formations superficielles quaternaires. Légende : 1. Indéterminé, 2. Moyen Âge, 3. Antiquité, 4. Protohistoire, 5. Néolithique, 6. Paléolithique, 7. Gisement certain, 8. Gisement probable, 9. Gisement douteux. DAO : T. Piau. Fonds orographique : RGE Alti $1 \mathrm{~m}$ de l'IGN, Fonds hydrographique : BD Carthage de l'IGN, Fonds Archéologique : BD Archéo du PCR ValEuRT, Système de coordonnées : Lambert 93 Conformal Conic.

préhistoriques par migration latérale des chenaux de l'Eure. Les enceintes/enclos circulaires non datés (Protohistoire ?) repérés en rive gauche, entre Marcilly-sur-Eure et Saussay, laissent supposer une relative stabilité de ce secteur durant I'Holocène récent, le cours de l'Eure s'étant déporté d'environ $1 \mathrm{~km}$ en rive droite à une période encore indéterminée. La préservation des paléoformes fluviales en rive gauche offre la possibilité de reconstituer et de dater les différentes phases de mobilité latérale de I'Eure au cours de la dernière période postglaciaire dans une perspective géoarchéologique. Cette portion de la vallée est encore peu urbanisée et la prédominance des champs cultivés favorise la découverte de gisements par prospection aérienne et pédestre. Dans la plaine d'inondation de l'Eure, les vestiges connus sont rattachés à I'Antiquité et au Moyen Âge. Les carrières d'extraction de granulats (ballastières entre Bueil et Breuilpont) et les potentielles phases d'alluvionnement au cours de I'Holocène récent ont pu détruire en partie, ou masquer localement, les indices d'occupation les plus fugaces des périodes pré- et protohistoriques (structures fossoyées, restes lithiques).

En ce qui concerne les gisements repérés en contexte de plateau, ceux de rive droite sont pour la plupart mis au jour dans des secteurs où l'épaisseur de la couverture lœssique est supérieure à $1 \mathrm{~m}$ au centre des plateaux (Bréval, Tilly, Longnes). En rebord de plateau et sur les hauts de versant où le substrat calcaire est affleurant, ils sont peu nombreux et postérieurs à I'Antiquité (Bueil, Chaussyd'Ivry). Dans cette position topographique, les processus d'érosion et de décapage intense (ruissellement et ravinement) ont probablement participé au démantèlement des vestiges pré- et protohistoriques. En rive gauche, le corpus archéologique est très pauvre sur les plateaux crayeux nappés 
de manteaux d'altérites (argiles à silex) et de biefs à silex. Les gisements sont localisés à proximité des vallées sèches d'Ezy et de Boissière en tête de vallon, dans des secteurs en pente faible. Cette lacune pourrait être liée à une insuffisance des recherches (biais scientifique) et/ou à une érosion du manteau d'altérites et des limons éoliens (biais taphonomique). Enfin, les sites implantés sur les versants sont plus rares. Ils sont repérés dans des têtes de vallon à faible pente (sous-bassin du Radon et vallées d'Ezy et de Boissière). Sur les versants, qui sont des zones de transport sédimentaire dominant caractérisés par des sols d'érosion jeunes (sols bruns lessivés tronqués), les processus de remaniement sont importants (solifluxion en contexte périglaciaire et érosion agricole durant I'Holocène) et la troncature des enregistrements archéologiques est fréquente [21]. En termes de potentiel archéologique, les versants orientés nord-ouest et nord-est avec des séquences lœssiques weichséliennes bien développées mais discontinues sont souvent propices à la découverte de vestiges paléolithiques (vallées sèches d'Ezy et de Boissière en rive gauche). Les glacis d'érosion des buttes stampiennes (argiles à meulières et sables stampiens) en rive droite de l'Eure sont des contextes favorables à la mise au jour de gisements préhistoriques bien préservés du fait d'un important colluvionnement le long des versants à l'origine de dépôts de pente stratifiés. L'association buttes stampiennes/occupations paléolithiques a d'ailleurs été mise en évidence à plusieurs reprises sur les plateaux de Brie à Sénart en Seine-et-Marne [22]. En contexte de plateaux lœssiques, la préservation des gisements archéologiques est souvent médiocre, surtout en rebord de plateau où la couverture limoneuse est peu épaisse. Néanmoins, la mise en culture rend les vestiges archéologiques plus facilement identifiables en prospection de surface en raison des importants remaniements liés aux labours. Cette configuration géomorphologique permet d'expliquer la forte concentration de gisements sur les plateaux en rive droite de I'Eure. Enfin, plusieurs questions émergent quant aux modalités de gestion de la ressource en eau, en particulier sur les plateaux de rive gauche où la forte perméabilité de la craie réduit la densité de drainage. L'approvisionnement en fer pendant la Protohistoire et I'Antiquité et l'exploi-

[21] BORDERIE \& CHAMAUX 2016.

[22] Blaser et AL. 2010 ; Chaussé et AL. 2015. tation ponctuelle des gisements ferreux dans les zones de Sables de Lozère et les bancs de meulières restent à étudier.

Ce premier état des lieux permet de confirmer, en plus de la variable « intensité de la prospection », le rôle majeur des processus géomorphologiques (ruissellement diffus et concentré, processus gravitaires) dans la préservation actuelle des gisements archéologiques. L'alluvionnement et le colluvionnement ont probablement participé à l'enfouissement et à la conservation des gisements (terrasses alluviales quaternaires entre Saussay et Anet, glacis colluviaux des buttes stampiennes). A contrario, en rive concave, en contexte de plateau lœssique ou sur les versants les plus pentus, les remaniements liés à la dynamique fluviale, au ruissellement diffus et aux pratiques agro-pastorales ont pu impacter l'intégrité des gisements et/ou les rendre plus facilement visibles en prospection de surface. La carte archéologique actuelle de la fenêtre d'étude ne reflète donc pas l'occupation réelle de l'espace par les sociétés anciennes, intégrant ainsi un certain nombre de biais taphonomiques individualisés à l'échelle régionale. À plus long terme, une analyse fine des formations superficielles (nature, géométrie, chronologie) devrait permettre de préciser les conditions d'évolution et de préservation des gisements archéologiques à une échelle plus locale.

\section{RECONSTITUTION PRÉLIMINAIRE DE L'ENVIRONNEMENT GÉOMORPHOLOGIQUE D'UN SANCTUAIRE D'ÉPOQUE ROMAINE DANS LA PLAINE D'INONDATION DE L'EURE}

En 2011, des prospections aériennes (association Archéo27) ont mis en évidence les traces d'un sanctuaire d'époque romaine dans la plaine d'inondation de l'Eure (rive droite) sur la commune de Garennessur-Eure au lieu-dit «Bellevue ». L'édifice, entouré d'un portique, adopte un plan rectangulaire, proche du carré, avec une galerie périphérique de même plan, caractéristique du type fanum (fig. 5A). La cartographie régionale des formations superficielles (cf. 4.1) couplée à l'analyse d'un modèle numérique de terrain à haute résolution (RGE alti $1 \mathrm{~m}$ ) a permis d'identifier à proximité immédiate de ce sanctuaire plusieurs paléoformes fluviales. Des prospections sur le terrain (pédestre et géophysique) ont confirmé l'existence d'une zone de paléochenal, bien marquée dans la topographie actuelle, d'environ $50 \mathrm{~m}$ de large et incisée de $2 \mathrm{~m}$ dans la grave grossière à silex roulés d'âge weichsélien. Le remblaiement diachrone de cette entité offre la possibilité de do- 
cumenter et dater les jalons de I'histoire paléoenvironnementale de l'Eure dans sa moyenne vallée en lien avec l'occupation humaine (fig. 5B, C et D). Ce site à fort potentiel géoarchéologique a été retenu comme zone atelier afin d'analyser, de façon croisée et à une échelle locale, les données archéologiques, historiques et géomorphologiques dans un objectif commun d'interpréter l'environnement du site archéologique (contexte topographique d'implantation) et d'éclairer les interrelations entre le sanctuaire antique de Bellevue et la plaine d'inondation de l'Eure (risques passés, exploitation et gestion de la ressource en eau).

L'étude des faciès sédimentaires dérivés des carottes exploratoires BEL I et BEL II permettent de proposer un premier séquençage du remblaiement alluvial. Le cadre chrono-stratigraphique reste toutefois à étayer. Un granoclassement normal décroissant est observé de la base du remblaiement de chenal (graviers et sables limoneux) à son sommet (limons sableux). Cette configuration révèle une perte graduelle de compétence. La base du remblaiement alluvial (unité I) est composée de sables graveleux interprétés comme le sommet de la charge de fond $d$ 'un chenal énergique. Succèdent ensuite des sables limoneux gris à intercalation de sables grossiers (unité II) qui traduisent des variations dans les conditions hydrodynamiques du chenal. L'unité III (sables limoneux gris à traits d'hydromorphie) indique le passage d'un chenal de type énergique à moribond. Ce dernier paraît se déconnecter progressivement du drain principal qui s'éloigne à une distance modérée (défluviation ou changement de style fluvial ?). L'unité IV est composée de limons sableux gris carbonatés riches en malacofaunes et micro-charbons de bois datés entre 2146 et 2010 av. J.-C. sur la carotte BEL II [23]. Le chenal, en cours d'atterrissement, n'est probablement plus en eau permanente durant cette période. Enfin, des dépôts d'inondation limono-sableux assez grossiers (entre 40 et $50 \mathrm{~cm}$ d'épaisseur, unité $V$ ) viennent napper le sommet du remblaiement alluvial. Ces dépôts de plaine d'inondation provoquent une uniformisation de la topographie du fond de vallée (Fig. 9). L'analyse morpho-sédimentaire et paléohydrologique préliminaire du chenal de Bellevue permet finalement d'identifier trois grandes phases : (1) mise en place d'un chenal énergique sans doute vers le milieu de I'Holocène ; (2) déconnexion du chenal de l'axe fluvial principal et remblaiement progressif à partir de l'âge du Bronze (2146-2010 av. J.-C.) par des limons sableux carbonatés probablement issus de la

[23] Le sédiment organique de I'unité IV du carottage BEL 2 a fait l'objet d'un prélèvement pour une datation AMS (BetaAnalytic). Un second échantillon avait également été prélevé sur I'unité IV de BEL I pour datation radiocarbone mais ce dernier n'a fourni aucun résultat.

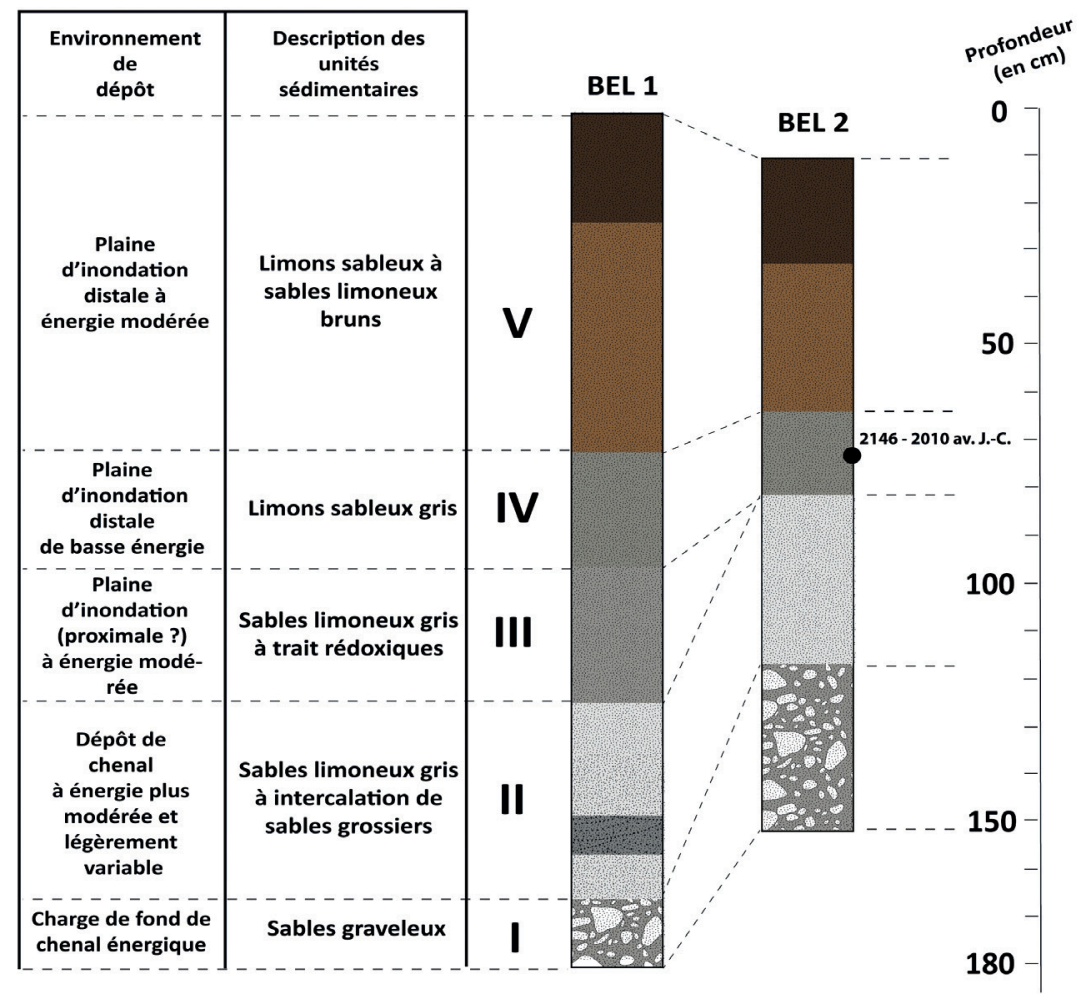

Figure 9

Interprétation en termes de milieux de sédimentation des unités sédimentaires décrites à partir des carottes BEL I et BEL I| réalisées dans le remblaiement du paléochenal de Bellevue. DAO : T. Piau (d'après PIAU 2019). 
dégradation des sols du bassin-versant de l'Eure ; (3) atterrissement et abandon total du chenal de I'Antiquité jusqu'à nos jours. Ces premiers résultats indiquent que si le chenal a bien été remblayé avant l'implantation du sanctuaire, le site antique est resté potentiellement soumis aux inondations de I'Eure lors de crues épisodiques.

\section{CONCLUSIONS ET PERSPECTIVES DE RECHERCHE}

La prospection géomorphologique s'est avérée fondamentale pour mettre en lumière la diversité des archives sédimentaires de l'Eure moyenne et le rôle des biais taphonomiques (érosion/sédimentation) dans la préservation des gisements archéologiques au sein de chaque compartiment du géosystème (plateau, versant, fonds de vallée et vallon). Elle a contribué à faire émerger deux zones ateliers possédant un fort potentiel paléoenvironnemental et géoarchéologique. Sur le site de Bellevue, l'étude géoarchéologique souligne le potentiel élevé des archives sédimentaires fluviatiles pour caractériser la trajectoire socio-environnementale de I'Eure moyenne au cours de I'Holocène et mettre en lumière les variables de contrôle dans cette évolution. L'année 2020 sera consacrée à la poursuite des campagnes de prospections géophysiques (TRE et géoradar) et de carottages autour du complexe cultuel où d'autres paléochenaux ont été individualisés. En complément des analyses sédimentologiques et des datations géochronologiques (OSL sur sables quartzeux, AMS et ${ }^{14} \mathrm{C}$ sur sédiments organiques et micro-charbons de bois), une étude des malacofaunes est envisagée afin de mieux caractériser les unités morpho-écologiques contemporaines de chaque milieu de sédimentation. Par ailleurs, le sous-bassin du Radon, affluent de I'Eure en rive droite, offre une documentation archéologique diachronique (enclos protohistoriques, sanctuaires d'époque romaine, nécropoles et habitats mérovingiens, commanderie médiévale) et un cadre morpho-sédimentaire favorable à la mise en œuvre d'une approche par budget sédimentaire. Une reconnaissance préliminaire de terrain a permis de cibler les secteurs clés pour analyser les profils pédologiques mis en place sur les dépôts lœssiques et reconstituer la géométrie des dépôts alluviaux dans le fond de vallée le long de transects représentatifs. L'objectif sera d'appréhender la temporalité des dynamiques érosives et de quantifier la redistribution des sédiments à différentes échelles spatio-temporelles. À plus longue échéance, d'autres prélèvements sédimentaires sont envisagés dans un sous-bassin à lithologie crayeuse de rive gauche de l'Eure : la vallée sèche d'Ezy.

Enfin, ce travail de recherche s'inscrit au cœur d'enjeux socio-culturels de premier ordre. La sauvegarde du patrimoine culturel de la vallée de I'Eure, notamment archéologique, menacé de disparaître du paysage (érosion, ouverture de carrières pour l'exploitation des granulats, urbanisation croissante) et de la mémoire collective apparaît comme un enjeu de conservation majeure. L'inventaire du patrimoine archéologique, dernier témoignage des interrelations Homme-milieu à l'échelle du Quaternaire, pourra contribuer à définir les futures stratégies de conservation et de valorisation de cet héritage culturel en tenant compte de l'impact des processus géomorphologiques passés et actuels. Parmi les sites à forte valeur géoscientifique et culturelle, on notera le gisement de Saint-Prest (haute terrasse de l'Eure à restes de mammifères et vestiges acheuléens) et la coupe de Chaudon (séquence de lœss-paléosols et vestiges préhistoriques du Paléolithique moyen [24]) qui font figure de référence pour la géologie du Quaternaire et la préhistoire de la vallée de l'Eure. D'autres sites plus confidentiels et/ou en cours d'étude (coupe de Guainville) méritent aussi une attention particulière et une réflexion collective en ce qui concerne leur sauvegarde. Mené en étroite collaboration avec les acteurs locaux (élus, gestionnaires, associations, habitants) et les collectivités (région, départements et leurs services dédiés : DRAC, services archéologiques), le programme de recherche ValEuRT veut agir comme un vecteur de diffusion des connaissances géoarchéologiques auprès de la population comme des acteurs locaux et, à travers les propositions de valorisation didactique des sites, contribuer à la mise en valeur socio-économique du territoire étudié (renouveler les circuits culturels et touristiques).

[24] BORDERIE 2014. 
BeAuchamp, Axel, 2019, Impacts des aménagements hydrauliques sur les systèmes fluviaux bas-normands depuis 2000 ans. Approches géomorphologique et géoarchéologique, Thèse de doctorat, Université de Caen Normandie.

Berger, Jean-François, Carozza, Jean-Michel, Castanet, Cyril, Cubizolle, Hervé, Dendievel, AndréMarie, Deschodt, Laurent, Franc, Odile, Ghilardi, Matthieu, Lespez, Laurent, Salvador, Pierre-Gil, Sivan, Olivier, Vannière, Boris, Argant, Jacqueline, Boulen, Muriel, Brochier, Jacques-Léopold, Carozza, Laurent, Germain-Vallée, Cécile \& Lemer, Laurence, 2018, « Climat et environnements : les étapes de la première anthropisation de l'espace (6 000-2 000 BCE) en France » dans Jean Guilaine \& Dominique Garcia (éd.) : La protohistoire en France, Paris (collection Histoire et Archéologie).

Bétard, François, Viel, Vincent, Arnaud-Fassetta, Gilles \& Piau, Théophile, 2020, « Géomorphologie et paléoenvironnements de la Vallée de l'Eure. Éléments contextuels et perspectives géoarchéologiques » dans Fabienne Dugast \& Ingrid Renault (éd.), Gestion et formation des territoires de la Préhistoire à nos jours. Approches et perspectives exploratoires, Montagnac (collection Archéologie du paysage).

Blaser, Frédéric, Blaser, Romana, Wuscher, Patrice \& Bruant, Jean, 2010, « Une occupation du Paléolithique moyen sur la butte des « Hauts-Fresnais » à Ballainvilliers (Essonne) », Revue Archéologique d'Tle-de-France 3, p. 5-22. BORDERIE, Quentin, 2014, QuOrEL. Inventaire documentaire des formations quaternaires d'origine éolienne en Eureet-Loir, Rapport de programme de prospection thématique, Décembre 2014, Conseil général d'Eure-et-Loir, Chartres.

Borderie, Quentin \& Chamaux, Gabriel, 2016, QuOrEL, formations limoneuses quaternaires d'origine éolienne en Eure-et-Loir : rapport 2015-2016, Conseil général d'Eure-et-Loir, Chartres.

Bravard, Jean-Paul \& MAgnY, Michel (éd.), 2002, Les fleuves ont une histoire. Paléoenvironnement des rivières et des lacs français depuis 15000 ans, Paris.

Brookes, Andrew, 1988, Channelized rivers. Perspectives for environmental management. Wiley, Chichester.

Brown, Anthony Gavin, 1997, Alluvial geoarchaeology. Floodplain archaeology and environmental change, Cambridge. Chaussé, Christine, Blaser, Frédéric, Debenham, Nick, Roque, Céline \& Vartanian, Emmanuel, 2015, «Pléistocène supérieur et paléolithique dans le domaine des sables stampiens (rupéliens) du sud du bassin de Paris : les données du site de Melun-Montaigu (Seine-et-Marne, France) », Quaternaire 26/3, p. 245-255.

Dewolf, Yvette, 1970, «Premières observations sur deux coupes de la vallée de l'Eure ». Bulletin de l'Association française pour l'étude du quaternaire 7/2-3, p. 191-198.

Dewolf, Yvette, 1974, Carte géomorphologique détaillée de la France au 1/50 000 : feuille XX-14, Saint-André-deI'Eure, Carte et notice explicative, CNRS, RCP 77, Paris.

DugAst, Fabienne, 2019, «Projet Vallée de I'Eure : une rivière, des territoires », Journée de restitutions des projets exploratoires OPUS 2017-2018, 6 juin 2019, Paris.

FencKe, Emilie, 2016 avec coll. Chamaux, Gabriel, Borderie, Quentin, DouARD, Michel, NoËL, Jean-Yves, PERRICHON, Pierre, SelLes, Hervé \& SOREL, Yoann, « Anet-Saussay (Centre - Eure-et-Loir), Liaison routière RD928-RD116. Des occupations de la Protohistoire ancienne à l'époque contemporaine : rapport de diagnostic d'archéologie préventive », Chartres, Conseil départemental d'Eure-et-Loir.

GilignY, François (éd.), 2005, Louviers «La Villette » (Eure) : un site du Néolithique moyen en zone humide, Rennes (Documents archéologiques de l'Ouest).

GoNet, Adrien, 2017, Du plateau au fond de vallée : Apport de l'étude de trois sites archéologiques à la compréhension des dynamiques géomorphologiques holocènes en Normandie, Thèse de doctorat, Université de Rouen Normandie. Guillemin, Claudine, 2017, «Les alluvions anciennes de l'Eure à Guainville », Inventaire du Patrimoine Géologique de la Région Centre, Fiche argumentaire de présélection des sites, DREAL Centre.

LACOMBE, Olivier \& OBERT, Daniel, 2000, « Héritage structural et déformation de couverture : plissement et déformations tertiaire dans l'Ouest du bassin de Paris », Comptes Rendus de l'Académie des Sciences 300, p. 793-798.

LEBLÉ, Geoffrey, 2016, « Une séquence sédimentaire plurimillénaire de l'Eure à Val de Reuil (27) 》, ArchéoThéma 38, p. 69.

LeSPEZ, Laurent, 2012, Le temps de l'environnement et des paysages des systèmes fluviaux au cours de l'Holocène. Normandie, Grèce, Mali, Mémoire d'Habilitation à Diriger des Recherches, 1, Université de Caen Basse-Normandie. MagnY, Michel, 2019. Aux racines de l'Anthropocène. Une crise écologique reflet d'une crise de I'homme, Lormont.

MoreaU, Clément, 2011. Val de Reuil, «Le Chemin aux Errants », Rapport final d'opération de diagnostic archéologique, Archeodunum.

Obert, Daniel, Deffontaines, Benoît \& Gely, Jean-Pierre, 1992, « Adaptation du réseau hydrographique aux structures et à l'évolution néotectonique. Application au Bassin Parisien », Bulletin d'Information des géologues du Bassin de Paris 29/4, p. 85-95. 
Obert, Daniel, Deffontaines, Benoît, Gely, Jean-Pierre, Joly, Michel, Lacombe, Olivier, Lorenz, Jacqueline \& POMERoL, Charles, 1997, « Tectonique plicative de couverture dans le Lutétien supérieur de la vallée de l'Eure : Indice d'un accident profond », Bulletin d'Information des géologues du Bassin de Paris 34/2, p. 13-18.

Pastre, Jean-François, Fontugne, Michel, Kuzucuoglou, Catherine, Leroyer, Chantal, LimondinLozouet, Nicole \& TALON, Marc, 1997, « L'évolution Tardi- et Postglaciaire des lits fluviaux au nord-est de Paris (France). Relation avec les données paléoenvironnementales et l'impact anthropique sur les versants », Géomorphologie : relief, processus, environnement 4, p. 291-312.

Pastre, Jean-François, Orth, Paul, Le Jeune, Yann \& Bensaadoune, Sonia, 2006, « L'homme et l'érosion dans le Bassin Parisien (France). La réponse morpho-sédimentaire des fonds de vallée au cours de la seconde partie de I'Holocène » dans Philippe Allée \& Laurent Lespez (éd.), L'érosion entre société, climat et paléoenvironnements. Actes de la table-ronde en I'honneur de René Neboit-Guilhot, Clermont-Ferrand (collection Nature et Société 3), p. 237-247.

Prau, Théophile, 2019, Dynamique géomorphologique holocène et occupation humaine dans le bassin-versant de I'Eure : apport d'une approche géoarchéologique multiscalaire, mémoire de Master 2, Université de Paris, Paris.

Reimer, Paula J., Bard, Edouard, Bayliss, Alex, BeCK, Warren J., Blackwell, Paul G., Bronk RaMsey, Christopher, Buck, Caitlin E., Cheng, Hai, Edward, Lawrence R., Friedrich, Michael, Grootes, Pieter M., Guilderson, Thomas P., Haflidason, Haflidi, Hajdas, Irka, Hatté, Christine, Heaton, Timothy J., Hoffman, Dirk, Hogg, Alan G., Hughen, Konrad A., KaIser, Felix K., Kromer, Bernd, Manning, Sturt W., Niu, Mu, Reimer, Ron W., Richards, David A., Scott, Marian E., Southon, John R., Staff, Richard A., Turney, Christian S. M., \& VAN Der Plicht, Johannes, 2013, « IntCal13 and Marine13 Radiocarbon Age Calibration Curves 0-50,000 Years cal BP », Radiocarbon 55/4, p. 1869-1887

Wuscher, Patrice, Blaser, Romana, Cammas, Cécilia, Durand, Juliette, Glisoni, Steve, Saron, Emmanuelle \& PASTRE, Jean-François, 2012, «Des plateaux, des limons et des hommes : état des connaissances et questions sur les sols et l'érosion tardiglaciaires et holocènes en Île-de-France », Revue Archéologique d'Ile-deFrance 5, p. 5-28. 